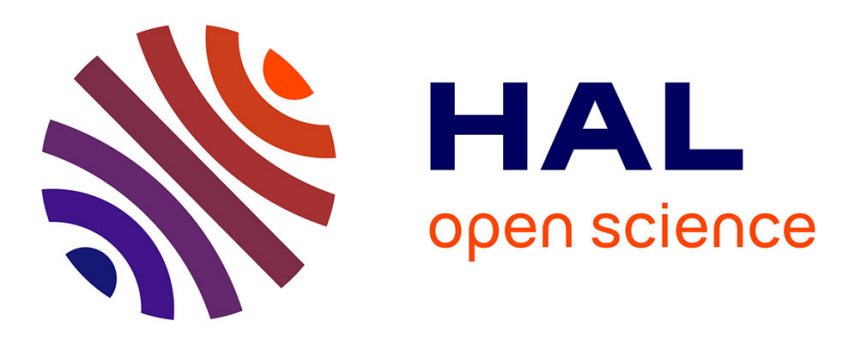

\title{
Rainbow guiding of the lowest-order antisymmetric Lamb mode in phononic crystal plate
}

Jinfeng Zhao, Weitao Yuan, Bernard Bonello, Bahram Djafari-Rouhani, Yongdong P Pan, Zheng Zhong

\section{- To cite this version:}

Jinfeng Zhao, Weitao Yuan, Bernard Bonello, Bahram Djafari-Rouhani, Yongdong P Pan, et al.. Rainbow guiding of the lowest-order antisymmetric Lamb mode in phononic crystal plate. Science in China Series E: Technological Sciences, 2019, 62 (3), pp.458-463. 10.1007/s11431-018-9398-y . hal-03046320

\section{HAL Id: hal-03046320 \\ https://hal.sorbonne-universite.fr/hal-03046320}

Submitted on 8 Dec 2020

HAL is a multi-disciplinary open access archive for the deposit and dissemination of scientific research documents, whether they are published or not. The documents may come from teaching and research institutions in France or abroad, or from public or private research centers.
L'archive ouverte pluridisciplinaire HAL, est destinée au dépôt et à la diffusion de documents scientifiques de niveau recherche, publiés ou non, émanant des établissements d'enseignement et de recherche français ou étrangers, des laboratoires publics ou privés. 


\title{
Rainbow guiding of the lowest-order antisymmetric Lamb mode in phononic crystal plate
}

\author{
ZHAO JinFeng $^{1 \dagger *}$, YUAN WeiTao $^{1 \dagger}$, BONELLO Bernard ${ }^{2}$, DJAFARI-ROUHANI \\ Bahram $^{3}$, PAN YongDong ${ }^{1} \&$ ZHONG Zheng ${ }^{4 *}$ \\ ${ }^{1}$ School of Aerospace Engineering and Applied Mechanics, Tongji University, 100 Zhangwu Road, 200092 Shanghai, China \\ ${ }^{2}$ Sorbonne Université, UPMC Université Paris 06, INSP UMR CNRS 7588, 4 Place Jussieu, 75005 Paris, France \\ ${ }^{3}$ Institut d'Electronique, de Microélectronique et de Nanotechnologie (IEMN-UMR CNRS 8520), Université de Lille Sciences et \\ Technologies, Cité Scientifique, 59652 Villeneuve d'Ascq Cedex, France \\ ${ }^{4}$ School of Science, Harbin Institute of Technology, Shenzhen, 518055, PR, China
}

${ }^{2}$ INSP UMR CNRS 7588, Sorbonne Université, UPMC Université Paris 06, Paris 75005, France

${ }^{3}$ Institut d'Electronique, de Microélectronique et de Nanotechnologie (IEMN-UMR CNRS 8520), Université de Lille Sciences et Technologies, Villeneuve d'Ascq Cedex 59652, France

\begin{abstract}
The modern acoustic circuits have experienced significant progress with the development of artificial structures, including phononic crystals, metamaterials or metasurfaces. Among the most intensive topics, the reconfigurable acoustic guides are achieved through several mechanisms. In this work, we report on the rainbow guiding for the lowest-order antisymmetric Lamb $\left(\mathrm{A}_{0}\right)$ mode in a phononic crystal plate, where the linear waveguides are constituted by erecting aligned pillars either solid or hollow. We show both numerically and experimentally that the whispering gallery modes (WGMs) in the hollow pillars can be generated by the defect mode that propagates in the waveguide. Then, the reemission of $\mathrm{A}_{0}$ mode by the WGMs to the outer lateral areas can lead to spatial control of frequency selective wave guidance called 'rainbow guiding'. Our approach allows for an accurate control of the wave guiding and facilitates the integration of waveguides within other devices in the acoustic circuits.
\end{abstract}

Keywords: whispering gallery mode, rainbow guiding, phononic crystal

\section{Introduction}

After having been the subject of intensive fundamental research [1-6], phononic crystals (PCs) are at the heart of several devices including sensors [2], acoustic lenses [3,4], diodes [5], and waveguides [6]. These latter are generally based on the existence of one or several defect modes created by introducing an accurately designed linear or L-shaped defect into an otherwise perfect PC [7-10]. Apart from this methodology, there have been some other prominent progress recently. Among them, one set of works are featured by using the topologically protected modes, such as valley modes, to guide wave along the path at the boundaries between two PCs [11-13]; another set focus on implementing resonators, for example the hollow pillars into a PC, in order to create high quality factor waveguides owing to the occurrence of whispering gallery modes (WGMs) in these resonators [14-16]. In view of the manifold

\footnotetext{
$\dagger$ ZHAO JinFeng and YUAN WeiTao contributed equally to this work.

* Corresponding authors (Email address: jinfeng.zhao@tongji.edu.cn, zhongzheng@ @it.edu.cn)
} 
polarization symmetries of WGMs and the constraints that may result from the requirement of an external wave source [15,16], the features of WGMs are not yet fully investigated. For instance, only the normally incident wave has been used and only one type of quadrupole WGM has been generated to date.

In parallel, the spatial control of frequency selective trapping for elastic waves has raised increasing attention. This issue was first investigated in optics and was known as 'rainbow trapping' [17-20]. It refers to stopping the different frequency component of light at a different location and the formation of a 'trapped rainbow'. In the acoustic field, the rainbow trapping has also been addressed by the graded structures [21,22], PCs [23], space-coiling metamaterials [24], or Mie resonance-based structures [25]. These structures can stop and trap acoustic waves of different frequencies at a different location, through the modulated group velocity [21,22] or the frequency cascade effect [25,26], by gradually changing the structure and/or material parameters along or normally to the propagating direction [20,21]. During this process, it is noticed that introducing resonators such as Mie resonators within the waveguide allows to tilt the propagating direction [25]. This finding helps open new possibilities of incorporating the rainbow trapping devices of waves with other devices, for example the PC based waveguides, in order to make use of the trapped energy. However, such combination of different devices is quite rarely reported. One of the major challenge is the mismatch between the resonant frequencies of resonators, in general in the low frequency range, and the operating frequency of the waveguide that falls usually in the high frequency range. Regarding tilting the wave direction, the quadrupole WGMs naturally transfer the trapped energy to a direction different the one that incident wave comes from. Meanwhile, the frequency of WGMs can fall within both the low and high frequency ranges [15]. These features make the WGMs a suitable candidate for providing a simple link between the rainbow trapping device and waveguide, for trapping and guiding waves of different frequencies at different position. To date, the combination of PC based waveguide, rainbow trapping effects and WGMs into one device has never been reported in the literature.

In this work, we investigate the guiding achievements of linear waveguides comprising hollow pillars at some places in their lateral edges. Based on both the existence of a defect mode in the waveguide and the quadrupole WGMs in the hollow pillars, we demonstrate both numerically and experimentally the spatial control of frequency selective guidance for the lowest-order antisymmetric Lamb $\left(\mathrm{A}_{0}\right)$ mode. We discuss also the impact of the generation of WGMs.

\section{Dispersion curves and whispering gallery mode}


(a)

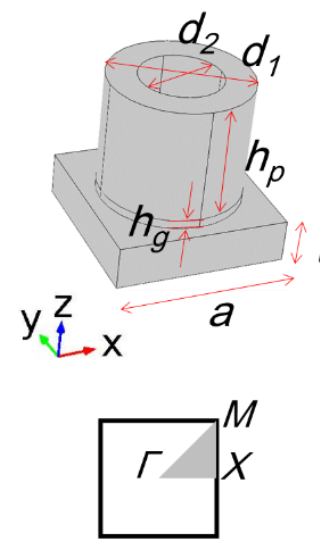

(b)

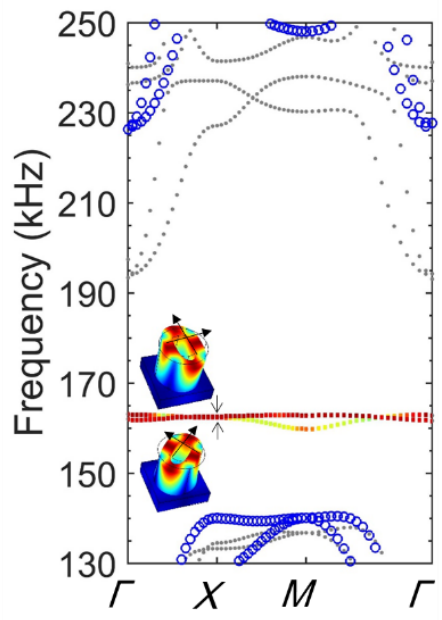

(c)

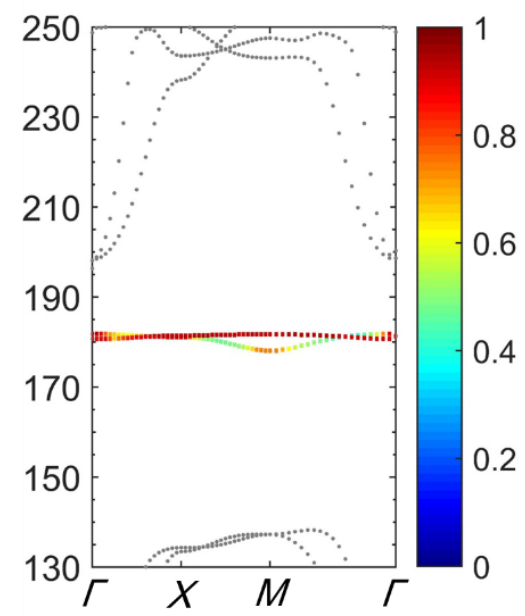

Figure 1 (a) Top panel: Schematic view of the square-latticed unit cell and the geometrical parameters.

Bottom panel: The reduced Brillouin zone (BZ). Band structure (grey dotted lines) of the hollow PC plate wherein $a=6, t=1.5, h_{g}=0.135, h_{p}=4, d_{1}=5$ and (b) $d_{2}=2.9$ or (c) $d_{2}=2.7 \mathrm{~mm}$. In Figure 1(b),

the blue circle lines represent the dispersion in the solid PC plate $\left(d_{2}=0\right)$, while inset shows the normalized distribution of total displacement $u_{t}$ and the modal shapes for WGMs at point $X$ of BZ. The colors in the WGMs branches correspond to the parameter $\alpha=\iiint_{\text {pillar }} u_{t} d V / \iiint_{\text {cell }} u_{t} d V$. The color bar in the right panel is for both $u_{t}$ and $\alpha$.

The guiding structures were constituted by solid or hollow steel pillars glued to an aluminum plate by epoxy adhesive at the outlet interface between the PC in a square lattice and the plate (see a general view in Figure 2(b) and (c)). The PC is designated below as hollow or solid PC according to whether it includes hollow or solid pillars in the unit. The top panel in Figure 1(a) shows the unit cell derived from the actual sample in experiment, whereby an upper steel pillar and a lower epoxy resin pillar are fixed on one side of the aluminum plate. The geometrical parameters are the lattice constant $a$, the plate thickness $t$, the height of the lower epoxy resin (the upper steel) pillar $h_{g}\left(h_{p}\right)$. Both the lower and upper pillars have the same outer and inner diameters, namely $d_{1}$ and $d_{2}$ respectively. We assume throughout this work that $a=6, t=1.5, h_{g}=0.135$ [27], and $d_{1}=5 \mathrm{in} \mathrm{mm}$. Regarding the physical properties of the different materials, namely steel, aluminum and epoxy resin we use Young's modulus (in GPa) 199, 67.7, 5.1, Poisson's ratio $0.25,0.35,0.38$, and the density (in $\left.\mathrm{g} \cdot \mathrm{cm}^{-3}\right) 7.85,2.7,1.2$, respectively. The bottom panel in Figure 1(a) shows the reduced Brillouin zone (BZ) for the square-latticed unit cell.

We use a finite element method (FEM) to compute the band structure of a solid PC plate $\left(d_{2}=0\right)$ when $h_{p}=4 \mathrm{~mm}$. A complete band gap opens up in between 140 and $226 \mathrm{kHz}$ as shown by the blue circle lines in Figure 1(b). We then have calculated the band structure of a hollow PC plate when $h_{\mathrm{p}}=4$ and $d_{2}=2.9$ or $2.7 \mathrm{~mm}$. The results are displayed as grey dotted lines in Figure 1(b) and 1(c) respectively. In contrast to the preceding case for the solid PC, two new flat branches appear in between 159.8 and $163.0 \mathrm{kHz}$ in Figure 1(b) or between 178 and $181.8 \mathrm{kHz}$ in Figure 1(c), because of the occurrence of quadrupole WGMs in the hollow pillars. Their modal shapes at point $X$ of the BZ are displayed by the inset in Figure 1(b) together with the polar axes noted by the black arrows, either parallel or featuring an angle of $\pm 45^{\circ}$ with respect to the axes $x$ and $y$. We will call them as WGM 1 and WGM 2 hereafter. 
The overlapped symbols whose color is defined by the ratio of the integrated total displacement $u_{t}$ in the steel and epoxy resin pillars against its counterpart in the unit cell $\alpha=\iiint_{\text {pillar }} u_{t} d V / \iiint_{\text {cell }} u_{t} d V$, presents the high concentration of elastic energy within the hollow pillar for both WGMs. The $u_{t}$ is calculated by $u_{t}=\sqrt{u_{x}^{2}+u_{y}^{2}+u_{z}^{2}}$, with $u_{x}, u_{y}$, and $u_{z}$ being the displacement along the $x$-, $y$-, and $z$-axes respectively. As shown by the inset in Figure 2(b), this high energy localization for WGMs must be ascribed to the fast decay of the displacement field from the top of the pillar towards its base. Due to this displacement field distribution, the coupling between the substrate plate and the pillar is relatively weak, so that the frequency position of WGMs depends mainly upon the properties of the hollow pillar, and is less affected by the lattice constant or symmetry of PC. Therefore, we could use the frequency of WGMs in the PC to represent the one in the single hollow pillar on the substrate plate.

\section{Physical model and numerical results}

After determining the frequency position for both the band gap and WGMs, we first build a symmetric structure (SS) as shown in Figure 2(b) where the linear waveguide is constituted by a path between two lines of pillars. The transverse width between the centers of the two rows of the guide is $2 a$ so that the energy flow within the channel is well assured for frequencies above $145 \mathrm{kHz}$ by the defect mode. On each lateral line, we erect at $x=5 a$ one hollow pillar with $d_{2}=2.9 \mathrm{~mm}$ (labeled 1, 2 in Figure 2(b) and 2(c)) and with $d_{2}=2.7 \mathrm{~mm}$ at $x=11 a$ (labeled 3,4); all the hollow pillars have the same height $h_{p}=4$ $\mathrm{mm}$ whereas the solid pillars have a height $h_{p}=5 \mathrm{~mm}$ in order to more efficiently confine the elastic energy and avoid any leakage out of the channel.

Let us consider the transversely uniform $\mathrm{A}_{0}$ Lamb mode incident upon the SS along the positive direction of the $x$ axis. Figure 2(a) shows the averaged transmissivity $T$ computed along a $2 a$ long horizontal line lying $3 \mathrm{~mm}$ above the hollow pillar 1 with $d_{2}=2.9 \mathrm{~mm}$ (red solid line), the hollow pillar 3 with $2.7 \mathrm{~mm}$ (blue dashed line) or the solid pillar located at $x=a$ (black solid line). It is noteworthy that all these calculations are performed using the finite element code COMSOL Multiphysics® in the frequency domain. Moreover, the aluminum substrate is bound to the perfectly matched layer (PML), so that the boundary reflections are eliminated. Figure 2(a) shows that the propagation above and below the solid pillars cannot happen since $T<0.1$ in a broad interval between 130 and $240 \mathrm{kHz}$; therefore, the confinement of waves within the guide is excellent. On the contrary, when measured next to a hollow pillar, a peak appears in the transmission spectrum at a frequency that well corresponds to the WGMs of the pillar. Actually, the $T_{\max }=0.37$ appears at $f=163 \mathrm{kHz}$ above the hollow pillar $1\left(d_{2}=\right.$ $2.9 \mathrm{~mm})$ whereas the $T_{\max }=0.39$ occurs at $f=181.5 \mathrm{kHz}$ above the hollow pillar $3\left(d_{2}=2.7 \mathrm{~mm}\right)$, which is in very good agreement with the frequencies of the WGMs displayed in Figure 1(b) and 1(c) respectively. In the vicinity of $130 \mathrm{kHz}$, there are other transmission peaks that shall be ascribed to the other modes in the band structures as shown in Figure 1(b) and 1(c), which are however out of the scope of this work. 


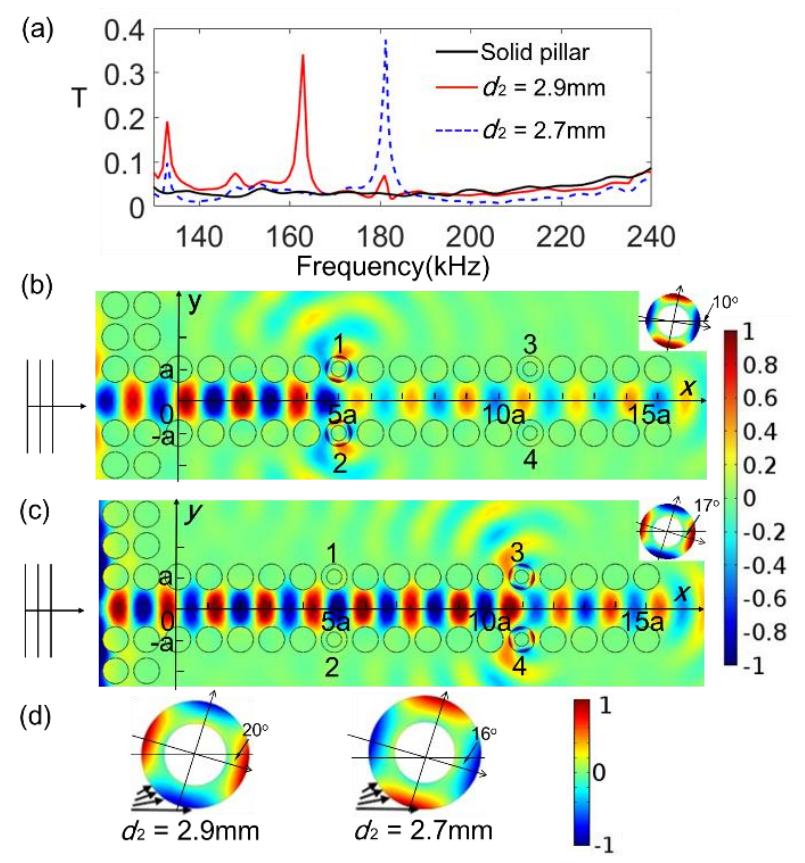

Figure 2 (a) Averaged $T$ of the symmetric structure computed along a horizontal line ( $2 a$ long) lying 3 $\mathrm{mm}$ above the hollow pillar with inner diameter $d_{2}=2.9 \mathrm{~mm}$ (red solid line), $2.7 \mathrm{~mm}$ (blue dashed line), or above the solid pillar (black solid line). Distribution of $u_{z}$ normalized to the amplitude of the incident wave when $f$ equals (b) $163 \mathrm{kHz}$ or (c) $181.5 \mathrm{kHz}$. Inset: zoom in of $u_{z}$ on top of the hollow pillar 1 or 3.

(d) Normalized distribution of $u_{z}$ on top of a hollow pillar glued on the aluminum plate with extra forces (black arrows) applied to the top outer edge when $f$ equals $163 \mathrm{kHz}$ (left) or $181.5 \mathrm{kHz}$ (right).

To give detailed information, Figures 2(b) and 2(c) show the amplitude of the out-of-plane displacement $u_{z}$ normalized to the amplitude of the incident wave, when $f=163$ and $181.5 \mathrm{kHz}$ respectively. Figure 2(b) highlights the defect mode propagating within the channel, the quadrupole WGM in the hollow pillars 1 and 2 (see also insets), and $\mathrm{A}_{0}$ Lamb mode reemitted to the homogeneous plate by the hollow pillars at resonance on WGM. Similar phenomena are noticed in Figure 2(c) except that the quadrupole WGM occurs in the hollow pillars 3 and 4 (see also insets). In Figure 2(b), the polar axes of the WGM are rotated by an angle of approximate $-10^{\circ}$ with respect to the coordinates system $(x, y)$, in between the respective orientations of WGM 1 and WGM 2. A similar trend is observable in Figure 2(c) but with a larger angle: the polar axes of WGM feature an angle of $-17^{\circ}$ with respect to the $x$ - or $y$ - axis. This can be understood from the relationship between the symmetry of the WGMs and the generation method. Actually, the incident $\mathrm{A}_{0}$ mode generates in the linear waveguide the transversely damping defect mode that impinges only a quarter of the outer perimeter of the hollow pillars 1 to 4 . Based on this analysis, we considered a single hollow pillar erected on the aluminum plate subjected to a field of forces on a quarter of the outer perimeter on the top surface (see black arrows in Figure 2(d)). The resultant normalized distribution of $u_{z}$ on pillar's top, as displayed by left and right panels in Figure 2(d) for the two considered geometries, shows good agreement with their respective counterpart in Figure 2(b) when $d_{2}=2.9 \mathrm{~mm}$ at $f=163 \mathrm{kHz}$ or in Figure 2(c) when $d_{2}=2.7$ $\mathrm{mm}$ at $f=181.5 \mathrm{kHz}$. Further calculations show that the polar axes of the generated WGM coincide with those of WGW 2 when an unit force is applied along the $x$ axis on a quarter of the top outer edge. It is therefore safe to say that both WGM 1 and WGM 2 are excited in the configuration described in Figure 2(b) and 2(c). Upon generation, the WGMs reemit the stored elastic energy to the external field 
in the form of $\mathrm{A}_{0}$ mode. It is noteworthy that the $T_{\max }$ displayed in Figure 2(a) is relatively small in comparison to the configuration where $\mathrm{A}_{0}$ mode impinges the hollow pillars at normal incidence with the $T_{\max }=0.7$ [15], because of the limited length of the arc-shaped source and the damping feature of the defect mode along $y$ axis. The value of $T_{\max }$ also helps understand the transmission at the outlet of the linear channel in Figure 2(b) and 2(c), although some incident wave is reflected back at the entrance of the channel [8]. Nevertheless, Figure 2(a)-2(c) demonstrate the spatial control of frequency selective guiding for $\mathrm{A}_{0}$ mode, also called "rainbow guiding".

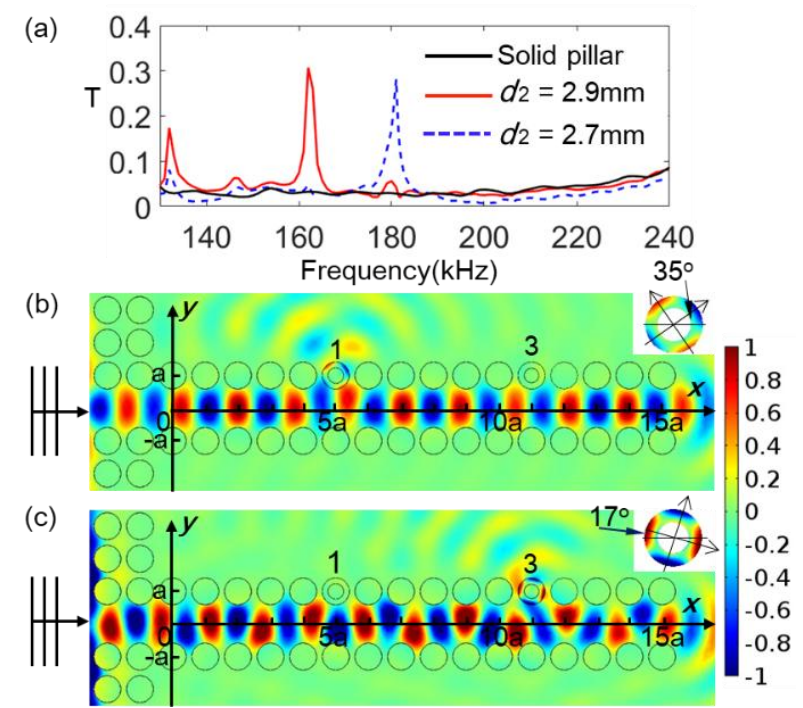

Figure 3 (a) Averaged $T$ of the asymmetric structure computed along a horizontal line ( $2 a$ long) lying $3 \mathrm{~mm}$ above the hollow pillar with inner diameter $d_{2}=2.9 \mathrm{~mm}$ (red solid line), $2.7 \mathrm{~mm}$ (blue dashed line), or behind the solid pillars (black solid line). Normalized distribution of $u_{z}$ when (b) $f=162 \mathrm{kHz}$ and (c) $f=181 \mathrm{kHz}$. Insets in (b) and (c) are the zoom in of $u_{z}$ on the top of the hollow pillars 1 and 3 respectively.

We built also another waveguide with the asymmetric structure (ASS) as shown in Figure 3(b). In this configuration the lower lateral side of the linear waveguide comprises only solid pillars while the upper side and the PC keep the same as before. We then calculated the averaged transmission coefficient $T$ for $\mathrm{A}_{0}$ mode incident normally on the ASS, at the same position as for the SS. The result is displayed in Figure 3(a) which shows that the transmission spectrum has quite similar characteristics as its counterpart in Figure 2(a): the $T<0.1$ behind the full pillars (black solid line), a transmission peak centered at $f=162 \mathrm{kHz}$ with $T_{\max }=0.33$ above hollow pillar 1 (red solid line), and another transmission peak centered at $f=181 \mathrm{kHz}$ with $T_{\max }=0.29$ above hollow pillar 3 (blue dashed line). The mappings of $u_{z}$ when normalized to the amplitude of the incident wave, are shown in Figure 3(b) and 3(c) at the frequencies when the $T_{\max }$ is reached. Obviously, both of these figures display the defect mode in the channel, the quadrupole WGM in hollow pillars 1 or 3 (see also inset), and $\mathrm{A}_{0}$ mode guided to the homogeneous plate through the hollow pillars. Therefore, the ASS also shows the rainbow guiding of $\mathrm{A}_{0}$ mode, being similar to the SS. In Figure 3(b) (resp. Figure 3(c)), the polar axes of WGM are rotated by an angle of $-35^{\circ}$ (resp. $-17^{\circ}$ ) with respect to the $(x, y)$ coordinates system, likewise what is observed with the SS in Figure 2(b) and 2(c). Our rainbow guiding device can be used in many fields. Of the noteworthy importance is the liquid sensor [14,16] when the hollow pillars are filled with different liquids, for which the guiding frequencies relate to $[14,16]$ the liquid density or thickness. 


\section{Experimental results}
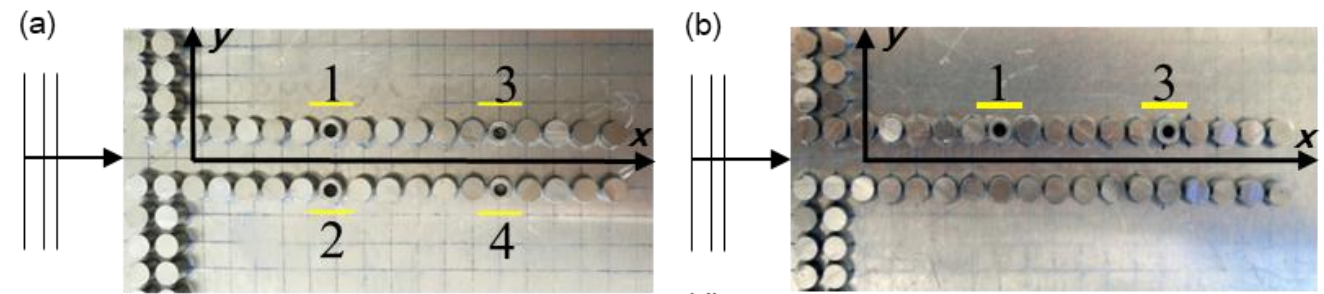

(c)

(d)
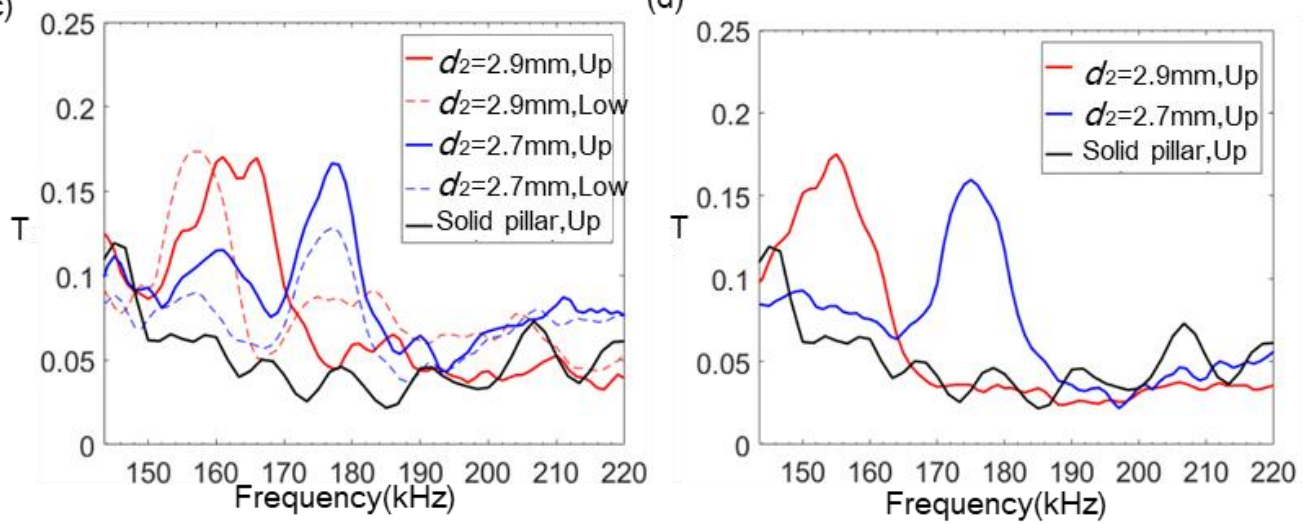

Figure 4 Top view of the (a) SS and (b) ASS samples. (c) Averaged $T$ for SS recorded along the horizontal line (in yellow in (a)) $3 \mathrm{~mm}$ above the hollow pillar 1 (red solid line) and below pillar 2 (red dashed line) with $d_{2}=2.9 \mathrm{~mm}$, or above the pillar 3 (blue solid line) and below pillar 4 (blue dashed line) with $d_{2}=2.7 \mathrm{~mm}$, or behind solid pillars (black solid line). (d) Averaged $T$ of the ASS (see (b)) mapped above hollow pillar 1 (red solid line), or pillar 3 (blue solid line), and behind solid pillars (black solid line).

We move in this last section to the experimental verification of the preceding findings. Figure 4(a) and 4(b) show respectively the manufactured SS and ASS samples whose geometrical parameters are in very good agreement with their nominal values. To excite and characterize the displacement field associated to the propagation of $\mathrm{A}_{0}$ mode, we used an unfocused air-coupled transducer that features the central frequency $f_{0} \approx 177 \mathrm{kHz}$ and the band width $\sim 32 \%$, for the excitation stage; and we adopted a laser ultrasonic scheme based on a laser Doppler vibrometer (LDV, Polytec vibrometer OFV 2570) to record $u_{z}$. Details on the technique are given in Ref [27,28]. The SS or ASS were attached to a very large aluminum plate so that the boundary effects could be ignored.

Figure 4(c) displays the coefficient $T$ averaged over the linear areas (yellow labels in Figure 4(a)) lying on the SS at the same positions as for the numerical study above. Behind the solid pillars (black solid profile) no transmission occurs since $T<0.1$ in the whole frequency range, namely $150-220 \mathrm{kHz}$, which is in agreement with the simulations. Above hollow pillar 1 (red solid line) or below hollow pillar 2 (red dashed line), two peaks centered at $f=160$ or $158 \mathrm{kHz}$ with the same $T_{\max }=0.17$ appear in the spectrum. Here again, we find a good agreement with the numerical results displayed in Figure 2(a) when $d_{2}=2.9 \mathrm{~mm}$, clearly supporting the wave guiding based on the WGMs. On the other hand, the coefficient $T$ measured above hollow pillar 3 or below pillar $4\left(d_{2}=2.7 \mathrm{~mm}\right.$ for both), as shown by the blue solid and dashed lines in order, gives rise to two peaks both centered at $177 \mathrm{kHz}$ with $T_{\max }=0.17$ and 0.13 respectively. Each peak is in good agreement with the numerical profile shown in Figure 2(a) when $d_{2}=2.7 \mathrm{~mm}$, and both of them are attributed again to the WGMs. 
Concerning the ASS, Figure 4(d) shows the coefficient $T$ recorded above the hollow pillar 1 (red solid line), above the hollow pillar 3 (blue solid line), or behind the full pillars (black solid line). Each profile is similar to its counterpart in Figure 4(c), and shows good agreement with the simulated results in Figure 3(a). Therefore, we experimentally observed the rainbow guiding for $\mathrm{A}_{0}$ mode by using either the SS or ASS. It should be noted that for both samples, the coefficients $T_{\max }$ characterized experimentally are smaller than their counterparts derived from simulations. We attribute these discrepancies to unavoidable imperfections of samples and energy absorption not accounted for in the simulations. Moreover, the experimental results suggest transmitted spots broader than the numerical ones, because of the broad frequency width of the elastic wave source and the possible leakage of confined modes in return [29].

\section{Conclusion}

In summary, the rainbow guiding of $\mathrm{A}_{0}$ mode through the SS and ASS waveguides is demonstrated both numerically and experimentally. In particular, the WGMs in the hollow pillars are numerically investigated to analyze the achievements in guiding $\mathrm{A}_{0}$ mode from the linear defect to the lateral regions of the sample outside the guide. The rainbow guiding is further tested and verified experimentally by the air-coupled method and laser ultrasonic technique. Besides, we have discussed the mechanism underlying the generation of WGMs polarized along different orientations. Our structures combine the widely used linear defect modes and the WGMs in hollow pillars, showing an accurate way for the spatial control of frequency selective guiding for $\mathrm{A}_{0}$ mode and other types of elastic waves in the future.

\section{Acknowledgements}

The authors gratefully acknowledge the financial support from the National Natural Science Foundation of China (Grant No. 11602174) and the Fundamental Research Funds for the Central Universities (Grant No. 2016KJ008).

\section{References}

1. Maldovan M. Sound and heat revolutions in phononics. Nature, 2013, 503: 209--217

2. Ke M, Zubtsov M, Lucklum R. Sub-wavelength phononic crystal liquid sensor. J Appl Phys, 2011, 110: 026101-1--026101-3

3. He Z, Cai F, Ding Y, et al. Subwavelength imaging of acoustic waves by a canalization mechanism in a two-dimensional phononic crystal. Appl Phys Lett, 2008, 93:

233503-1--233503-3

4. Zhao J, Bonello B, Becerra L, et al. Focusing of Rayleigh waves with gradient-index phononic crystals. Appl Phys Lett, 2016, 108: 221905-1--221905-4

5. Li X F, Ni X, Feng L, et al. Tunable unidirectional sound propagation through a sonic-crystal-based acoustic diode. Phys Rev Lett, 2011, 106: 084301-1--084301-4

6. Celli P, Gonella S. Manipulating waves with LEGO® bricks: A versatile experimental platform for metamaterial architectures. Appl Phys Lett, 2015, 107: 081901-1--081901-5

7. Otsuka P H, Nanri K, Matsuda O, et al. Broadband evolution of phononic-crystal-waveguide eigenstates in real- and k-spaces. Sci Rep, 2013, 3: 3351-1--3351-5

8. Benchabane S, Gaiffe O, Salut R, et al. Guidance of surface waves in a micron-scale phononic crystal line-defect waveguide. Appl Phys Lett, 2015, 106: 081903-1--081903-4 
9. Baboly M G, Raza A, Brady J, et al. Demonstration of acoustic waveguiding and tight bending in phononic crystals. Appl Phys Lett, 2016, 109: 183504-1--183504-4

10. Dong H W, Wang Y S, Zhang C. Inverse design of high-Q wave filters in two-dimensional phononic crystals by topology optimization. Ultrasonics, 2017, 76: 109--124

11. Lu J, Qiu C, Ye L, et al. Observation of topological valley transport of sound in sonic crystals. Nat Phys, 2016, 13: 369-1--369-7

12. Yves S, Fleury R, Lemoult F, et al. Topological acoustic polaritons: Robust sound manipulation at the subwavelength scale. New J Phys, 2017, 19: 075003-1--075003-9

13. Yu S Y, He C, Wang Z, et al. Elastic pseudospin transport for integratable topological phononic circuits. Nat Commun, 2018, 9: 3072-1--3072-8

14. Wang Y F, Wang T T, Wang Y S, et al. Reconfigurable phononic-crystal circuits formed by coupled acoustoelastic resonators. Phys Rev Applied, 2018, 8: 014006-1--014006-8

15. Jin Y, Fernez N, Pennec Y, et al. Tunable waveguide and cavity in a phononic crystal plate by controlling whispering-gallery modes in hollow pillars. Phys Rev B, 2016, 93: 054109-1--054109-8

16. Jin Y, Pennec Y, Pan Y, et al. Phononic crystal plate with hollow pillars connected by thin bars. J Phys D: Appl Phys, 2017, 50: 035301-1--035301-8

17. Gan Q, Fu Z, Ding Y J, et al. Ultrawide-bandwidth slow-light system based on THz plasmonic graded metallic grating structures. Phys Rev Lett, 2008, 100: 256803-1--256803-4

18. Noda S, Chutinan A, Imada M. Trapping and emission of photons by a single defect in a photonic bandgap structure. Nature, 2000, 407: 608--610

19. Park J, Kim K Y, Lee I M, et al. Trapping light in plasmonic waveguides. Opt Express, 2010, 18 : $598--623$

20. Hu H, Ji D, Zeng X, et al. Rainbow trapping in hyperbolic metamaterial waveguide. Sci Rep, 2013, 3: 1249-1--1249-6

21. Zhu J, Chen Y, Zhu X, Garcia-Vidal F J, et al. Acoustic rainbow trapping. Sci Rep, 2013, 3: 1728-1--1728-6

22. Colombi A, Ageeva V, Smith R J, et al. Enhanced sensing and conversion of ultrasonic Rayleigh waves by elastic metasurfaces. Sci Rep, 2017, 7: 6750-1--6750-9

23. Tian Z, Yu L. Rainbow trapping of ultrasonic guided waves in chirped phononic crystal plates. Sci Rep, 2017, 7: 40004-1--40004-7

24. Ni X, Wu Y, Chen Z G, et al. Acoustic rainbow trapping by coiling up space. Sci Rep, 2014, 4: 7038-1--7038-6

25. Zhou C, Yuan B, Cheng Y, et al. Precise rainbow trapping for low-frequency acoustic waves with micro Mie resonance-based structures. Appl Phys Lett, 2016, 108: 063501-1--063501-5

26. Jiménez N, Romero-García V, Pagneux V, et al. Rainbow-trapping absorbers: Broadband, perfect and asymmetric sound absorption by subwavelength panels for transmission problems. Sci Rep, 2017, 7: 13595-1--13595-12

27. Zhang D, Zhao J, Bonello B, et al. Investigation of surface acoustic wave propagation in composite pillar based phononic crystals within both local resonance and Bragg scattering mechanism regimes. J Phys D: Appl Phys, 2017, 50: 435602-1--435602-10

28. Zhang D, Zhao J, Bonello B, et al. Air-coupled method to investigate the lowest-order antisymmetric Lamb mode in stubbed and air-drilled phononic plates. AIP Adv, 2016, 6: 085021-1--085021-8 
29. Marchal R, Boyko O, Bonello B, et al. Dynamics of confined cavity modes in a phononic crystal slab investigated by in situ time-resolved experiments. Phys Rev B, 2012, 86:

224302-1--224302-5 